\title{
Induced Remodeling of Porcine Tendons to Human Anterior Cruciate Ligaments by $\alpha-G A L$ Epitope Removal and Partial Cross-Linking
}

\author{
Kevin R. Stone, MD, Ann Walgenbach, RN, ${ }^{1}$ and Uri Galili, $\mathrm{PhD}^{2}$
}

This review describes a novel method developed for processing porcine tendon and other ligament implants that enables in situ remodeling into autologous ligaments in humans. The method differs from methods using extracellular matrices (ECMs) that provide postoperative orthobiological support (i.e., augmentation grafts) for healing of injured ligaments, in that the porcine bone-patellar-tendon-bone itself serves as the graft replacing ruptured anterior cruciate ligament (ACL). The method allows for gradual remodeling of porcine tendon into autologous human ACL while maintaining the biomechanical integrity. The method was first evaluated in a preclinical model of monkeys and subsequently in patients. The method overcomes detrimental effects of the natural anti-Gal antibody and harnesses anti-non-gal antibodies for the remodeling process in two steps: Step 1. Elimination of $\alpha$-gal epitopes - this epitope that is abundant in pigs (as in other nonprimate mammals) binds the natural anti-Gal antibody, which is the most abundant natural antibody in humans. This interaction, which can induce fast resorption of the porcine implant, is avoided by enzymatic elimination of $\alpha$-gal epitopes from the implant with recombinant $\alpha$-galactosidase. Step 2. Partial cross-linking of porcine tendon with glutaraldehyde - this cross-linking generates covalent bonds in the ECM, which slow infiltration of macrophages into the implant. Anti-non-gal antibodies are produced in recipients against the multiple porcine antigenic proteins and proteoglycans because of sequence differences between human and porcine homologous proteins. Anti-non-gal antibodies bind to the implant ECM, recruit macrophages, and induce the implant destruction by directing proteolytic activity of macrophages. Partial cross-linking of the tendon ECM decreases the extent of macrophage infiltration and degradation of the implant and enables concomitant infiltration of fibroblasts that follow the infiltrating macrophages. These fibroblasts align with the implant collagen fibers and secrete their own collagen and other ECM proteins, which gradually remodel the porcine tendon into human ACL. This ligamentization process lasts $\sim 2$ years and the biomechanical integrity of the graft is maintained throughout the whole period. These studies are the first, and so far the only, to demonstrate remodeling of porcine tendon implants into permanently functional autologous ACL in humans.

Keywords: anterior cruciate ligament implants, porcine tendon, anti-Gal, anti-non-gal, xenotransplantation, $\alpha$-galactosidase

\section{Introduction}

$\mathbf{P}$ orcine eXtracellular matrix (ECM) implants of decellularized tissues and organs are the subject of extensive research for biocompatible replacement of tissues with impaired function in humans. ${ }^{1,2}$ Most porcine
ECMs studied in the area of ligament repair in orthopedic surgery are "patches" or sheets of connective tissue ECMs that are attached around the injured ligament to provide orthobiological support, which decreases the in vivo mechanical forces on the ligament repair during postoperative healing, prevents repair gap formation or failure, and

\footnotetext{
${ }^{1}$ The Stone Clinic and Foundation, San Francisco, California.

${ }^{2}$ Department of Surgery, University of Massachusetts Medical School, Worcester, Massachusetts (retired).
}

(C) Kevin R. Stone et al. 2017; Published by Mary Ann Liebert, Inc. This Open Access article is distributed under the terms of the Creative Commons Attribution Noncommercial License (http://creativecommons.org/licenses/by-nc/4.0/) which permits any noncommercial use, distribution, and reproduction in any medium, provided the original author(s) and the source are credited.

*This article is part of a special focus issue on Strategic Directions in Musculoskeletal Tissue Engineering. Additional articles can be found in Tissue Engineering Part A, volume 23, numbers 15-16. 
improves host cell infiltration for the regeneration of the injured tissue. $^{3-7}$

We have been interested in determining whether the porcine ligament tissue itself can be used as an implant that undergoes remodeling and reconstruction into a functioning autologous ligament after its grafting to replace ruptured ligament in humans. In this review, we describe studies that led to the successful development of a novel method for processing of porcine bone-patellar-tendon-bone implants, which enables the in situ conversion of the porcine tendon grafts into functional autologous human anterior cruciate ligaments (ACLs) while being constantly subjected to biomechanical stress. The preclinical and clinical studies described in this review on ACL reconstruction by porcine tendon further suggest that processed porcine tendons and ligaments may be used for reconstruction of other torn ligaments and tendons in orthopedic surgeries.

\section{Treatments for Ruptured ACLs}

Rupture of the ACL is a common injury. More than 200,000 damaged ACLs undergo surgical intervention in the United States each year. The two techniques currently practiced in such surgical interventions are bone-patellartendon-bone ligament autograft harvested from the knee for reconstructing the ACL, or cadaveric bone-patellar-tendonbone allografts. After the implantation of autograft or allograft tendon, the implant undergoes a process of remodeling called "ligamentization". ${ }^{8-12}$ In this process, the fibroblasts within the implant become necrotic because of ischemia and macrophages infiltrating the implant debride the necrotic tissue and induce vascularization of the implant. Fibroblasts of the recipient repopulate the implant and align with the collagen fibers' "scaffold." These fibroblasts secrete collagen and other ECM proteins ultimately resulting in gradual remodeling of implant into an autologous tissue with characteristic ACL structure.

The harvest of an autograft patellar tendon from the knee often results in increased pain, because of a second surgical site, longer recovery periods, and increased morbidity. Complications may further include patella fracture, patellar tendon rupture, and scar formation. ${ }^{12,13}$ Cadaveric tendon allografts offer a limited source of ACL replacement tissue because of scarcity of available tissue from young, healthy donors. Allografts from elderly cadavers might be of significant lower biomechanical capacity than allograft from young cadaveric donors. The perceived risk of transmission of hepatitis and other infectious diseases is another obstacle to the acceptance of cadaveric tissue.

In view of the disadvantages and risks in use of tendon autografts or allografts for ACL reconstruction, we have explored the possible use of porcine patellar tendon implants that are transplanted according to the methods using autologous or allogeneic tendon for ACL reconstruction. If successful, porcine patellar tendon may serve as a limitless source of implants of standardized age and size thereby assuring quality of the implant, as it will be harvested from pigs of optimal age. In addition, sterility of the graft will be controlled by rigorous sterilization.

\section{Considerations in Using Porcine Tendon Implants in Humans}

The immune system in nonhuman primates and in humans reacts against porcine implant grafts, except for grafts con- structed of collagen, a protein with low immunogenicity. ${ }^{14-16}$ This immune response induces extensive infiltration of macrophages that cause proteolytic degradation of the implant. ${ }^{16}$ To enable in situ gradual remodeling and regeneration of porcine tendon into autologous human ACL, while maintaining biomechanical function of the graft, the anti-implant immune response in the recipient has to be attenuated. Such attenuation is required to slow the immune-mediated destruction of the implant. In addition, the ligament implant fibers, comprising collagen and other ECM proteins, should be partially cross-linked to retain the biomechanical capacity of the ligament implant throughout the period of ligamentization, that is, destruction of the implant and its concomitant repopulation and regeneration by the recipient's fibroblasts that produce the autologous ECM. Such cross-linking would further slow the implant destruction by slowing the infiltration of macrophages, thereby allowing for fibroblasts to follow these macrophages, align with the porcine collagen fibers, and secrete autologous ECM for the remodeling of the implant. As detailed hereunder, attenuation of the immune response to the porcine graft was achieved by incubation of the porcine tendon in a solution of recombinant $\alpha$-galactosidase followed by partial cross-linking with glutaraldehyde.

\section{Preclinical Studies in Monkeys}

As a first stage in this research, we used rhesus monkeys as the experimental primate model. Our objectives have been to determine in this model the optimal conditions for engineering pig tendon to be used for ACL reconstruction to further perform a clinical trial for evaluating safety and efficacy of such implants in patients with ruptured ACL. The rhesus monkey was chosen as a model because it produces the natural anti-Gal antibody like humans. ${ }^{17}$

\section{Anti-Gal response in implant recipients}

Anti-Gal is the most abundant natural antibody in humans, comprising $\sim 1 \%$ of circulating immunoglobulins ${ }^{18-21}$ and binding specifically to a mammalian carbohydrate antigen called the $\alpha$-gal epitope with the structure Gal $\alpha 1-3 \mathrm{Gal} \beta 1-$ 4GlcNAc-R. ${ }^{22}$ This epitope is abundant on glycolipids, glycoproteins, and proteoglycans of nonprimate mammals, including pigs, prosimians, and New World monkeys. ${ }^{17,23,24}$ In contrast, the $\alpha$-gal epitope is completely absent in Old World monkeys, apes, and humans; instead, these primates and humans produce the natural anti-Gal antibody in large amounts. ${ }^{17,19,23,24}$ As many as $1 \%$ of human B cells are capable of producing anti-Gal. ${ }^{25}$ Most of these B cells (designated anti-Gal B cells) are quiescent. ${ }^{25}$ In contrast, anti-Gal B cells along the gastrointestinal tract continuously produce anti-Gal against carbohydrate antigens presented on bacteria of the normal gastrointestinal flora. ${ }^{26,27}$ Similar to other nonprimate mammals, the $\alpha$-gal epitope is present in large amounts in most pig tissues, including patellar tendons where it is found as $\sim 1 \times 10^{11}$ epitopes $/ \mathrm{mg}$. ${ }^{28}$

Several studies demonstrated in primates the immune response against loose connective tissue ECM such as swine intestinal submucosa (SIS) ${ }^{29}$ and decellularized dermis. ${ }^{30}$ For determining anti-Gal response in monkeys against porcine tendon, an untreated porcine tendon with one bone plug was implanted across the knee joint of a rhesus monkey to replace the autologous ACL. The porcine tendon was implanted 
within tunnels drilled in the femur and tibia, anchored in the femur end by the bone plug, and sutured at the free end to the tibia. ${ }^{31}$ Monitoring the immune response in rhesus monkey recipient demonstrated a $\sim 1000$-fold increase in anti-Gal titer within 2 weeks, as measured by ELISA with synthetic $\alpha$-gal epitopes linked to bovine serum albumin ( $\alpha$-gal BSA) as solid-phase antigen (Fig. 1). ${ }^{31}$ This elicited anti-Gal response results from activation of the many quiescent anti-Gal B cells by glycoprotein-linked $\alpha$-gal epitopes released from the implant. A similar extensive anti-Gal response was observed in humans receiving xenograft cells expressing $\alpha$-gal epitopes, even under strong immunosuppression. ${ }^{32,33}$ The combined activities of the natural and elicited anti-Gal antibodies exacerbate the detrimental effects of this antibody because of its effective binding to the multiple $\alpha$-gal epitopes in the porcine implant, rapid recruitment of macrophages by complement chemotactic factors generated after anti-Gal $/ \alpha$ gal epitopes interaction, ${ }^{34,35}$ and binding of macrophages to the $\mathrm{ECM}$ after $\mathrm{Fc} / \mathrm{Fc}$ receptor interaction ${ }^{36}$ with anti-Gal bound to the implant. ${ }^{37}$ Macrophages further bind through their $\mathrm{C} 3 \mathrm{~b}$ receptors to $\mathrm{C} 3 \mathrm{~b}$ complement deposits on $\mathrm{ECM}^{38}$ Macrophages infiltrating into xenogeneic ECM implants were shown to release proteases that degrade the implant. ${ }^{39}$ Within the untreated porcine tendon implant, multiple recruited macrophages mediated its complete resorption within a period of 2 months (Fig. 1). ${ }^{31}$ A similar elevation of anti-Gal activity and anti-Gal-mediated resorption of implanted orthopedic tissue was observed in monkeys implanted with porcine cartilage containing $\alpha$-gal epitopes. $^{40-42}$ Thus, we concluded that a prerequisite for successful implantation of porcine dense connective tissue ECM implants in humans is the elimination of $\alpha$-gal epitopes from the implant. This conclusion is supported by observations demonstrating a higher efficacy of porcine dermis ECM implanted in monkeys when the ECM was devoid of $\alpha$-gal epitopes, in comparison with porcine dermis ECM expressing $\alpha$-gal epitopes. ${ }^{30}$ It should be noted that studies with SIS implants in monkeys and in anti-Galproducing mice demonstrated no detrimental effects of anti-Gal on implantation of SIS whether or not the SIS had

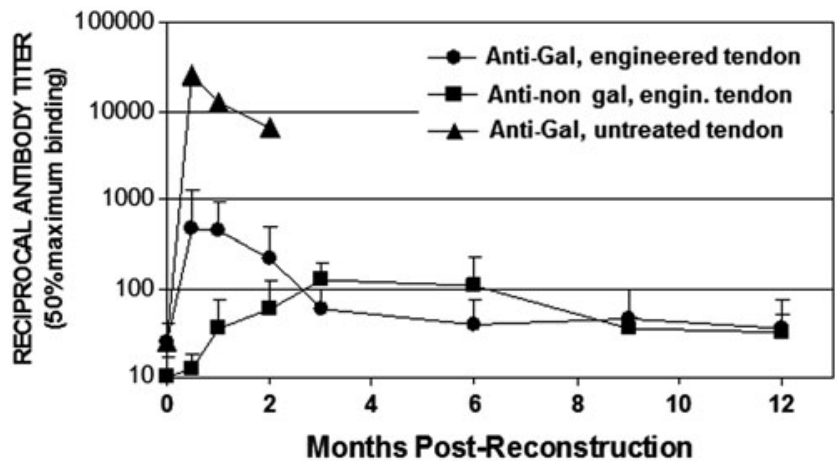

FIG. 1. Anti-Gal and anti-non-gal IgG titers in rhesus monkeys implanted with porcine tendon for ACL reconstruction. $\boldsymbol{\Delta}$, anti-Gal after implantation of untreated porcine tendon; •, anti-Gal after implantation of engineered tendon; घ, anti-non-gal after implantation of engineered tendon. Error bars represent standard deviation of reciprocal titers (modified from Ref., ${ }^{31}$ with permission). ACL, anterior cruciate ligament. $\alpha$-gal epitopes. ${ }^{14,43,44}$ The reason for these differences is the very low concentration of $\alpha$-gal epitopes on SIS. Because of relatively "great distance" between such epitopes on SIS, the detrimental effects of anti-Gal seem to be marginal. However, the high concentration of $\alpha$-gal epitopes in porcine tendons is detrimental upon implantation and requires the removal of this carbohydrate antigen.

Complete elimination of $\alpha$-gal epitopes from the dense connective tissue of porcine ligaments was achieved by incubation for $12-14 \mathrm{~h}$ in a solution of recombinant $\alpha$-galactosidase. ${ }^{28} \mathrm{~A}$ similar effective elimination of $\alpha$-gal epitopes by recombinant $\alpha$-galactosidase was demonstrated with porcine cartilage. ${ }^{42}$ The gene for this enzyme was cloned from coffee beans and expressed in yeast Pichia pastoris expression system. ${ }^{45,46} \mathrm{Re}-$ combinant $\alpha$-galactosidase cleaves the terminal galactosyl of the $\alpha$-gal epitopes, thus preventing subsequent binding of anti-Gal to the remaining portion of the carbohydrate chain with the structure Gal $\beta 1-4$ GlcNAc-R. ${ }^{28}$

Anti-Gal response in monkeys implanted with porcine tendon depleted of $\alpha$-gal epitopes and partially cross-linked with glutaraldehyde (see next section) was $\sim 100$-fold lower than that in the recipient of the untreated tendon (Fig. 1). The 10- to 20-fold increase in anti-Gal activity, observed after 2 weeks, is the result of an immune response against $\alpha$ gal epitopes on red cells and bone marrow cells enclosed within cancellous bone cavities in the bone plug, which were not accessible to $\alpha$-galactosidase. Glycoproteins carrying $\alpha$ gal epitopes that were released from these cells stimulated quiescent anti-Gal B cells to produce this antibody. ${ }^{28}$ Most of this anti-Gal response diminishes after 3 months because, by that time, the bone plug of the implant is almost completely remodeled into autologous bone and thus the source of glycoproteins with $\alpha$-gal epitopes is eliminated. ${ }^{28}$

\section{Cross-linking of tendon tissue with glutaraldehyde}

$\alpha$-Galactosidase-treated tendons further underwent partial cross-linking by glutaraldehyde to strengthen the biomechanical capacity of the tendon implant, thus avoiding tearing during in situ remodeling of the implant. ${ }^{28,31}$ Glutaraldehyde molecule has two aldol groups- $\mathrm{CHO}\left(\mathrm{CH}_{2}\right)_{3} \mathrm{CHO}$ which covalently interact with amino groups of proteins and thus crosslinks between proteins. These covalent bonds function as an obstacle, slowing the infiltrating macrophages. Glutaraldehyde cross-linking is widely used in processing of porcine heart valves for replacement of functionally impaired human heart valves. Incubation of porcine heart valves for days in $0.25 \%$ glutaraldehyde results in maximum cross-linking that blocks any infiltration of macrophages and of other inflammatory cells into the implanted heart valve. Therefore, heart valve implants do not undergo remodeling. Because of the extensive cross-linking, macrophages do not penetrate the implanted porcine valves and the detrimental deposits of these macrophages accumulate on the surface of the valve leaflets. ${ }^{47,48}$

It was reasonable to assume that, to enable remodeling of porcine tendon implants into autologous ACL, the crosslinking should be only partial, at a level that slows the destruction of implants but does not completely prevent infiltration of macrophages into the implant. ${ }^{28,31}$ This macrophage infiltration is required for allowing the gradual destruction of the implant at a pace slow enough to allow the recipient's fibroblasts to follow these macrophages. These 
infiltrating fibroblasts are expected to align with the porcine collagen fibers and secrete their collagen, thereby remodeling the porcine ligament implant into an autologous ACL. Optimal cross-linking was determined empirically by incubation of $\alpha$-gal-devoid patellar tendons in various concentrations of glutaraldehyde solution for $12 \mathrm{~h}$. Subsequently, the tendons were washed and incubated for $24 \mathrm{~h}$ in a $0.1 \mathrm{M}$ glycine solution, to block any free active aldol groups of glutaraldehyde. ${ }^{31}$

Tendons lacking $\alpha$-gal epitopes, incubated in $0.01 \%$ glutaraldehyde, were washed and implanted as ACL replacement in rhesus monkeys. Histological study of the implants after 2 months indicated that they were filled with large number of macrophages that induced extensive destruction of the xenograft. In contrast, tendons incubated in $0.25 \%$ glutaraldehyde displayed only marginal infiltration of macrophages, insufficient for remodeling, because of very high cross-linking. However, incubation in $0.1 \%$ glutaraldehyde was found to result in optimal levels of macrophage infiltration; therefore, this concentration was chosen for use in engineering of porcine tendon for implantation. ${ }^{31}$ The processed tendons were incubated with glycine for $24 \mathrm{~h}$, irradiated by $17.8 \mathrm{kGy}$ of e-Beam for final sterilization, and stored frozen until implantation. In vitro biomechanical stress tests indicated that this cross-linking of porcine tendons did not affect their biomechanical characteristics (unpublished observations).

\section{Implantation studies in monkeys}

A study was performed in 20 rhesus monkeys to evaluate the feasibility of the pig tendon devoid of $\alpha$-gal epitopes and partially cross-linked as an ACL reconstruction implant. ${ }^{31}$ Testing involved a unilateral monkey ACL reconstruction model with 2, 6, and 12 month sacrifice time points and clinical, histological, and biomechanical assessments. Monkeys receiving an allograft tendon for ACL reconstruction served as control. All animals returned to normal function by 7 weeks postoperatively. Range of motion and laxity were assessed by manual manipulation and comparison with contralateral, unoperated limbs. All range of motion and laxity measurements performed after 6 and 12 months were clinically acceptable, considering the surgical and anatomical complexities of the small primate knee. No clinically significant differences between allograft and treated porcine implants were noted in either clinical end point. ${ }^{31}$

In addition to anti-Gal activity measurements already described, serology studies assessed anti-non-gal antibodies production by the monkey recipients at various time points. These were measured by ELISA in which the solid-phase antigen was a homogenate of porcine tendon containing microscopic size fragments that was dried and thus adhered to ELISA wells as $2 \mathrm{mg} / \mathrm{mL}$. The serum in this assay was depleted of anti-Gal before the assay by adsorption on glutaraldehyde-fixed rabbit red cells. ${ }^{28}$ Anti-non-gal antibody activity peaks 3-6 months postimplantation because of the immune response against the multiple immunogenic proteins of the pig implant (Fig. 1). ${ }^{31}$ This immunogenicity is caused by the many differences in amino acid sequences between most homologous proteins in pigs and primates. These differences result in production of antibodies, called anti-non-gal antibodies, against most porcine proteins. ${ }^{15,16}$ The activity of these antibodies decreases by 9 and 12 months because much of the original porcine tissue causing immunological stimulation for production of anti-non-gal antibodies was eliminated at that time (Fig. 1). ${ }^{31}$

Necropsy in recipients of the processed porcine tendon implant demonstrated no degenerative articular cartilage changes in operative limbs, normal synovium healing, and normal ligament morphology. ${ }^{31}$ Histological evaluation of implants in both rhesus monkeys implanted with engineered porcine tendon and in control recipients of rhesus monkey allograft ligament revealed remodeling, leading to a gradual replacement of the initially engrafted matrix with host fibroblast and collagen, as well as advanced implanted tendon and bone integration in host bone tunnels. Postmortem ex vivo biomechanics analysis of the implants indicated that the ligamentized implants were not significantly different compared with the primate allograft cohort and compared favorably with various published values for autograft reconstructions in animals. The ligamentized-treated implants at both 6 and 12 months ${ }^{31}$ either equaled or exceeded the published strength of these autograft reconstructions performed in various animal models including primates. ${ }^{49-52}$ These observations in monkeys prompted us to perform a clinical trial on replacement of ruptured ACL with engineered porcine bone-patellar- tendon-bone implants.

\section{Implantation of Engineered Porcine Tendons in Patients}

Porcine patellar tendons were treated for $12 \mathrm{~h}$ with $\alpha$-galactosidase, washed, partially cross-linked for $12 \mathrm{~h}$ with $0.1 \%$ glutaraldehyde, washed, blocked with $0.1 \mathrm{M}$ glycine, and sterilized by irradiation with e-Beam (Fig. 2). These engineered tendons were studied as implants in patients who ruptured their ACL. This Phase I clinical trial was performed as an Food and Drug Administration (FDA)-approved singlecenter feasibility clinical study of the treated xenograft in 10 subjects in accordance with U.S. FDA Investigational Device Exemption and good storage practice requirements. ${ }^{28}$ The study protocol was approved by an institutional review board

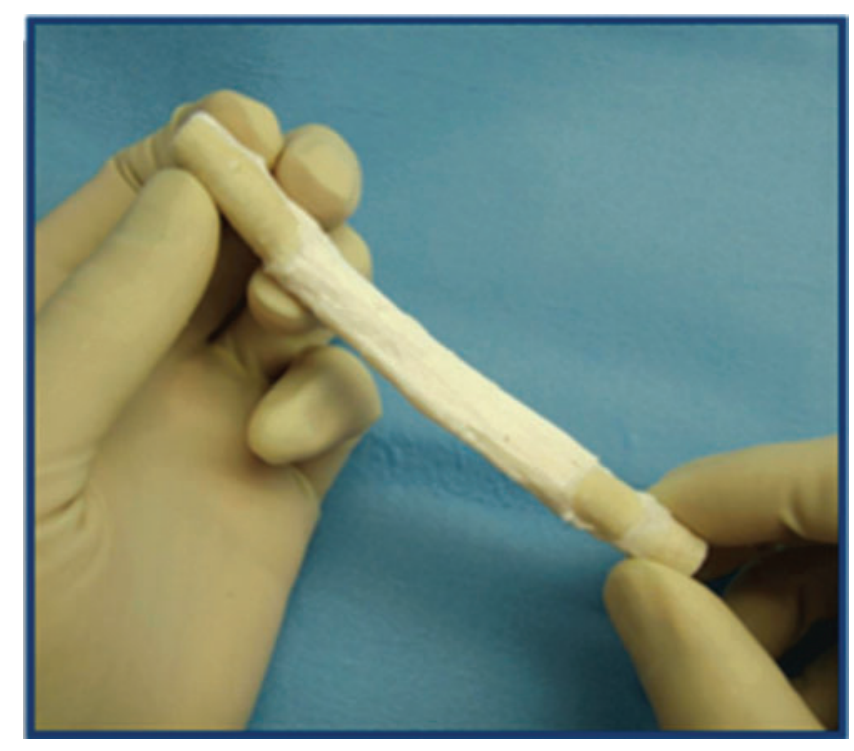

FIG. 2. Engineered ligament derived from porcine bonepatellar-tendon-bone implant. 
A

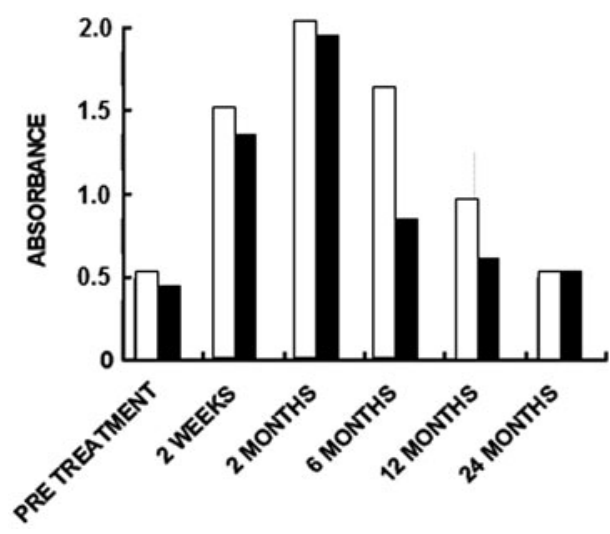

B Anti-non gal antibody

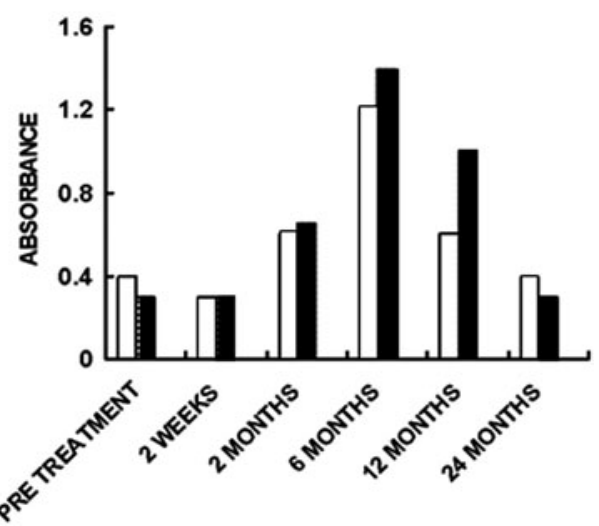

FIG. 3. Anti-Gal (A) and anti-non-gal IgG antibody response (B) in two representative orthopedic patients implanted with engineered pig patellar tendon for the replacement of ruptured ACL. Patient 1, open columns; Patient 2, closed columns. Anti-Gal antibody activity was determined by ELISA with $\alpha$-gal linked to bovine serum albumin as solid-phase antigen. Anti-non-gal antibody activity was determined with anti-Gal-depleted sera (adsorption on glutaraldehyde-fixed rabbit red cells), by ELISA with homogenate of fragmented pig tendon as solid-phase antigen. The figure shows antibody binding at serum dilution of 1:640 (based on data presented in Ref. ${ }^{28}$ ).

and all subjects provided written informed consent. The study objectives were verification of graft implantability, technical feasibility, and safety.

Of six evaluable subjects, five presented with functional implants at the 24-month postoperative time point and satisfied all study success criteria, including effusion, KT-1000, Pivot Shift, Lachman's and Anterior Drawer tests, as well as all safety parameters. ${ }^{28}$ The remaining subject, who was the only serious adverse event determined to be graft related, presented with tibia bone plug loosening at 15 months post ACL reconstruction and had his implant removed and tibial tunnel grafted with cancellous patellar tendon allograft. Four subjects were nonevaluable because of nondevice-related complications during the study because of trauma and very early return to sports. ${ }^{28}$ None of the patients displayed any indication of toxicity parameters in complete blood count (CBC), blood chemistry, and serology, as well as in urine chemistry at the 24-month time point of the study.

Analysis of anti-Gal IgG antibody activity demonstrated low increase that peaked at 2 months. This could be best demonstrated by comparison of anti-Gal binding to $\alpha$-gal BSA in ELISA in sera diluted 1:640, assayed at the various time points. ${ }^{28}$ Anti-Gal activity in two representative patients is presented in Figure 3A. On average, the preimplantation titer of anti-Gal was 1:200, and 2 months postimplantation, it increased only to $\sim 1: 1000$, then 6 months postimplantation, it decreased to $\sim 1: 700$ (not shown). This low increase in anti-Gal response reflects the activation of quiescent antiGal B cells by glycoproteins carrying $\alpha$-gal epitopes released from porcine bone marrow cells and red blood cells within the cancellous bone cavities of the implant. Anti-Gal returned to preimplant range by 12 months, reflecting the completion of the bone blocks remodeling process of the porcine bone into human bone. Anti-non-gal IgG response appeared after 2 months, peaked at $\sim 6$ months, and resolved to preimplant range by 24 months (Fig. 3B). ${ }^{28}$ These observations suggest that at $\sim 2$ years postimplantation, the porcine tissue within the implant completed the ligamentization process and was replaced by nonimmunogenic host fibroblasts, producing collagen fibers and other ECM components.

The diversity of anti-non-gal antibodies in tendon implant recipients could be demonstrated by Western blot analysis.

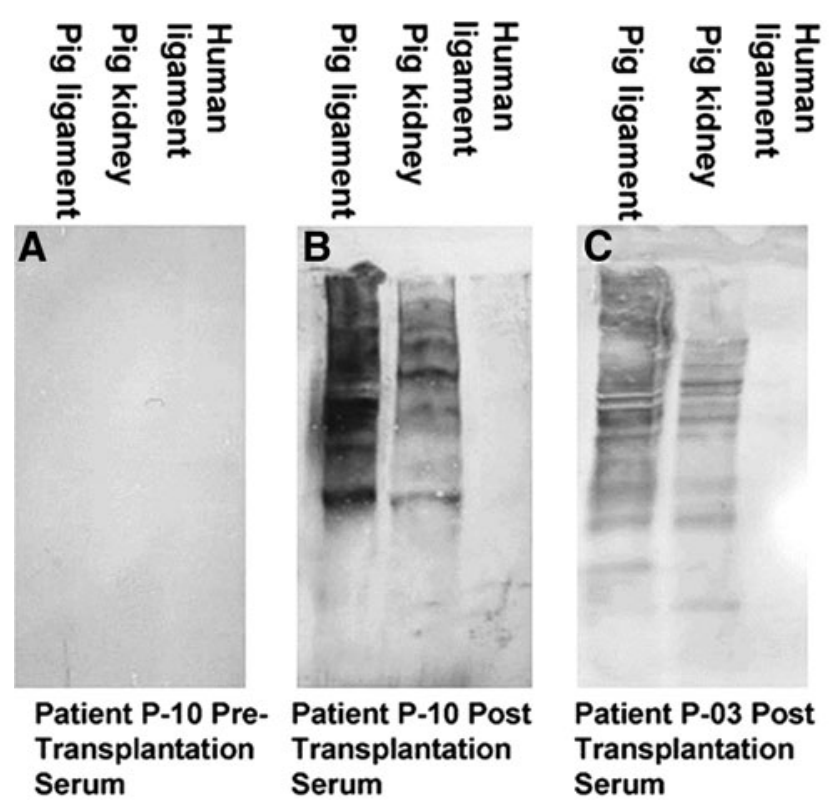

FIG. 4. Anti-non-gal antibody analysis by Western blots with porcine patellar tendon and kidney proteins or human tendon proteins separated on sodium dodecyl sulfatepolyacrylamide gel electrophoresis. (A) Preimplantation serum of patient P-10. (B) Serum of patient P-10, 6 months postimplantation. (C) Serum of patient P-03, 6 months postimplantation. In this analysis, the sera were depleted of anti-Gal (as shown in Fig. 3) and were diluted 1:10 (modified from Ref., ${ }^{28}$ with permission). 

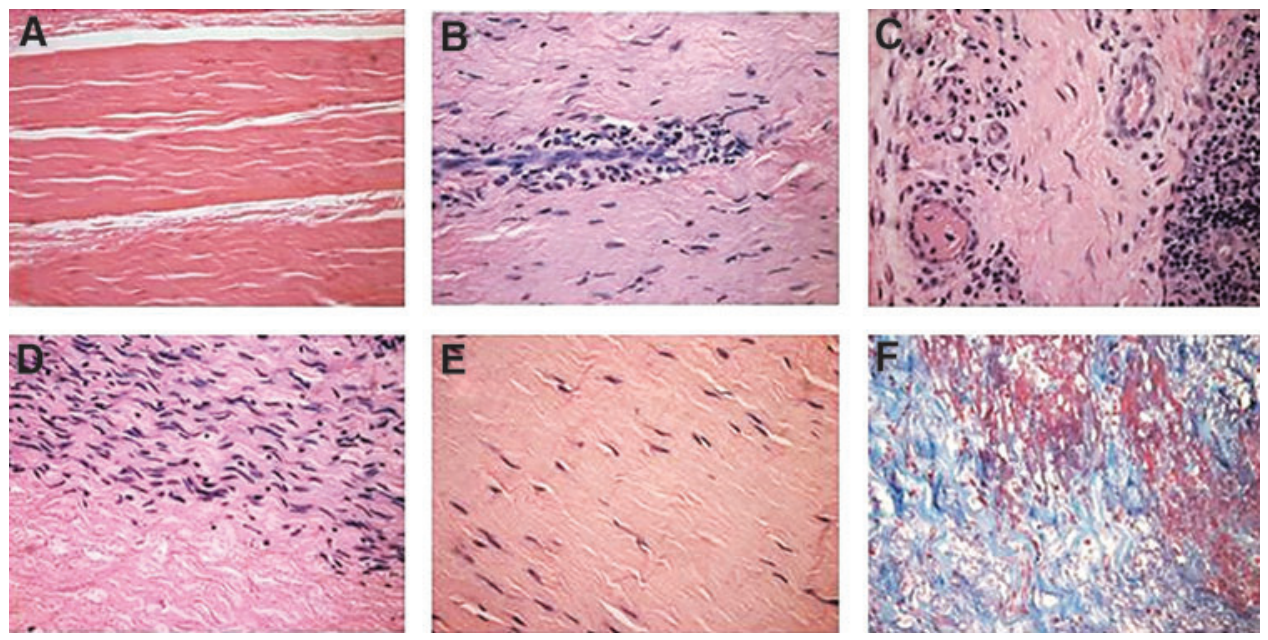

FIG. 5. Histological demonstration of various ligamentization stages in patients implanted with processed porcine tendon. (A) Porcine patellar tendon after processing for removal of $\alpha$-gal epitopes and cross-linking by glutaraldehyde. (B) Infiltration of macrophages into the implant. (C) Vascularization of the implant in a region near macrophage infiltrates. (D) Repopulation of a section of the implant by fibroblasts that aligned with the collagen fibers. (E) An advanced stage of ligamentization in which the repopulating fibroblasts are far apart from each other because of the collagen fibers and other matrix proteins and proteoglycans that have been secreted by these cells. (F) Collagen fibers of the implant stained blue by trichrome staining. (A-E): Hematoxylin and eosin, $(\mathbf{F})$ : trichrome $(\times 200)$ (from Ref., ${ }^{28}$ with permission).

Preimplantation and 6-month postimplantation sera were depleted of anti-Gal antibody and studied for binding to porcine tendon proteins separated by sodium dodecyl sulfate-polyacrylamide gel electrophoresis and blotted on nitrocellulose paper. ${ }^{28}$ As shown in Figure 4A, the patients had no measurable antibody binding to pig tendon proteins in the preimplantation sera. However, multiple antibodies bound to a large variety of porcine tendon proteins, creating a smear pattern rather than individual lines, were observed in postimplantation sera (Fig. 4B, C). These smear patterns are the result of partial overlap between bands of proteins of close size. Many of the porcine tendon proteins binding anti-non-gal antibodies were also observed in Western blot proteins of porcine kidney (Fig. 4B, C). ${ }^{28}$ The demonstration of these multiple antibodies is in accord with the notion that most pig proteins are immunogenic in humans because of $3-40 \%$ differences in the amino acid sequences between pig and humans. ${ }^{15}$ These anti-non-gal antibodies are highly specific to porcine proteins as indicated by the complete lack of antibody binding to human ligament proteins (Fig. 4B, C). ${ }^{28}$

Partial cross-linking of porcine tendons enabled conversion of anti-non-gal antibodies from antibodies inducing rapid macrophage mediated destruction of untreated tendon tissue into beneficial antibodies that mediate gradual remodeling of the pig tendon implant into an autologous ACL. The rapid destruction of untreated tendon, or of tendons under suboptimal processing, could be inferred from the extensive infiltration of macrophages that destroy prematurely the implant after cross-linking with $0.01 \%$ glutaraldehyde. ${ }^{31}$ In tendon implants that were cross-linked with the optimal glutaraldehyde concentration of $0.1 \%$, anti-non-gal antibodies binding to the tendon proteins also were likely to recruit macrophages by activating the complement system and generating complement cleavage chemotactic factors, as in most antigen-antibody interactions. The recruited mac- rophages further bind to the Fc portion of the anti-nongal antibodies immunocomplexed to the implant, secrete their proteases, and thus cause degradation of the porcine ECMs. However, because of the cross-linking, penetration of macrophages is slowed, enabling fibroblasts that follow the infiltrating macrophages to align with the porcine scaffold of collagen fibers and secrete their own ECMs, as shown in Figure 5. ${ }^{28}$ Ligamentization of the implant into human ACL, within a period of $\sim 2$ years, results in formation of a nonimmunogenic autologous ACL and thus, it may be functional for life without being subjected to a chronic anti-non-gal immune response. Indeed, long-term monitoring of the five evaluable patients has indicated that the porcine ligament implants are continuing to function as autologous ACL for $>10$ years.

The ligamentization process could be histologically observed in porcine tendon implants that were explanted from five of the patients, by secondary surgical intervention, due primarily to sport injuries (Fig. 5). ${ }^{28}$ Processed porcine tendons mainly contain the collagen fibers bioscaffold and the associated matrix proteins and proteoglycans (Fig. 5A). As already indicated, anti-non-gal antibodies binding to the implant are likely to contribute to the observed infiltration of macrophages into the transplanted porcine tendon by generating gradients of chemotactic complement cleavage peptides such as C5a and $\mathrm{C} 3 \mathrm{a}$ (Fig. 5B). These infiltrating macrophages also secrete cytokines, ${ }^{34}$ including vascular endothelial growth factor (VEGF), which induce vascularization of the implant (Fig. 5C). Recipient's fibroblasts infiltrate the implant through newly formed blood vessels and align with the porcine collagen fibers scaffold (Fig. 5D). These fibroblasts secrete their own collagen fibers and thus convert the implanted tendon into a viable autologous ACL (Fig. 5E). The collagen fibers are further stained blue in trichrome staining (Fig. 5F). To our knowledge, these studies are the first, and so far the only, to demonstrate remodeling of porcine tendon implants into functional permanent autologous ACL in humans. 


\section{Conclusions}

Porcine tendon and ligaments may be used as implants that undergo remodeling to become autologous ligaments in humans, if the immune response against such implants is attenuated. Porcine bone-patellar- tendon-bone served as a model in reconstruction of ruptured ACL. The porcine tendons were processed for removal of $\alpha$-gal epitopes by recombinant $\alpha$-galactosidase, followed by partial crosslinking with glutaraldehyde. Studies in rhesus monkeys demonstrated that implantation of such processed tendons, for replacement of resected ACL, resulted in gradual ligamentization of the implant into autologous ACL. Subsequent studies in humans demonstrated a similar process of remodeling of the tendon implant into autologous ACL within a period of $\sim 2$ years, while the biomechanical integrity is maintained. It is suggested that other porcine ligaments and tendons undergoing similar processing may be considered as implants in humans as well.

\section{Disclosure Statement}

The authors have received stock and consulting payments associated with the company Aperion Biologics, Inc. commercializing products referred to in this article.

\section{References}

1. Atala, A. Engineering organs. Curr Opin Biotechnol 20, 575, 2009.

2. Badylak, S.F., Weiss, D.J., Caplan, A., and Macchiarini, P. Engineered whole organs and complex tissues. Lancet 379, 943, 2012.

3. Valentin, J.E., Badylak, J.S., McCabe, G.P., and Badylak, S.F. Extracellular matrix bioscaffolds for orthopaedic applications. A comparative histologic study. J Bone Joint Surg Am 88, 2673, 2006.

4. Chen, J., Xu, J., Wang, A., and Zheng, M. Scaffolds for tendon and ligament repair: review of the efficacy of commercial products. Expert Rev Med Devices 6, 61, 2009.

5. Longo, U,G., Lamberti, A., Maffulli, N., and Denaro, V. Tendon augmentation grafts: a systematic review. Br Med Bull 94, 165, 2010.

6. Wisbeck, J.M., Parks, B.G., and Schon, L.C. Xenograft scaffold full-wrap reinforcement of Krackow Achilles tendon repair. Orthopedics 35, 331, 2012.

7. Xu, H., Sandor, M., Qi, S., Lombardi, J., Connor, J., McQuillan, D.J., and Iannotti, J.P. Implantation of a porcine acellular dermal graft in a primate model of rotator cuff repair. J Shoulder Elbow Surg 21, 580, 2012.

8. Butler, D.L. Anterior cruciate ligament: its normal response and replacement. J Orthop Res 7, 910, 1989.

9. Frank, C., Ameil, D., Woo, S., and Akeson, W. Normal ligament properties and ligament healing. Clin Orthop Relat Res 196, $15,1985$.

10. Noyes, F.R., Mooar, L.A., Moorman, C.T., and McGinniss, S.H. Partial tears of the anterior cruciate ligament. Progression to complete ligament deficiency. J Bone Joint Surg Br 71, 825, 1989.

11. Strickland, S.M., MacGillivray, J.D., and Warren, R.F. Anterior cruciate ligament reconstruction with allograft tendons. Orthop Clin North Am 34, 41, 2003.
12. Davarinos, N., O'Neill, B.J., and Curtin, W. A brief history of anterior cruciate ligament reconstruction. Adv Orthop Surg 2014, ID 706042, 2014.

13. Kohn, D., and Sander-Beuermann, A. Donor-site morbidity after harvest of a bone-tendon-bone patellar tendon autograft. Knee Surg Sports Traumatol Arthrosc 2, 219, 1994.

14. Daly, K.A., Stewart-Akers, A.M., Hara, H., Ezzelarab, M., Long, C., Cordero, K., et al. Effect of the $\alpha$-Gal epitope on the response to small intestinal submucosa extracellular matrix in a nonhuman primate model. Tissue Eng Part A 15, 3877, 2009.

15. Galili, U. Induced anti-non gal antibodies in human xenograft recipients. Transplantation 93, 11, 2012.

16. Galili, U. Avoiding detrimental human immune response against mammalian extracellular matrix implants. Tissue Eng Part B Rev 21, 231, 2015.

17. Galili, U., Clark, M.R., Shohet, S.B., Buehler, J., and Macher, B.A. Evolutionary relationship between the anti-Gal antibody and the Gal $\alpha 1 \rightarrow 3 \mathrm{Gal}$ epitope in primates. Proc Natl Acad Sci U S A 84, 1369, 1987.

18. Galili, U., Rachmilewitz, E.A., Peleg, A., and Flechner, I. A unique natural human IgG antibody with anti- $\alpha-$ galactosyl specificity. J Exp Med 160, 1519, 1984.

19. Teranishi, K., Manez, R., Awwad, M., and Cooper, D.K. Anti-Gal $\propto 1-3 \mathrm{Gal}$ IgM and IgG antibody levels in sera of humans and old world non-human primates. Xenotransplantation 9, 148, 2002.

20. Parker, W., Lin, S.S., Yu, P.B., Sood, A., Nakamura, Y.C., Song, A., Everett, M.L., and Platt, J.L. Naturally occurring anti- $\alpha$-galactosyl antibodies: relationship to xenoreactive anti- $\alpha$-galactosyl antibodies. Glycobiology 9, 865, 1999.

21. Hamadeh, R.M., Galili, U., Zhou, P., and Griffis, J.M. Human secretions contain $\operatorname{IgA}$, IgG and IgM anti-Gal (anti- $\alpha$ galactosyl) antibodies. Clin Diagnos Lab Immunol 2, 125, 1995.

22. Galili, U., Macher, B.A., Buehler, J., and Shohet, S.B. Human natural anti- $\alpha$-galactosyl IgG. II. The specific recognition of $\alpha(1-3)$-linked galactose residues. J Exp Med 162, 573, 1985.

23. Galili, U., Shohet, S.B., Kobrin, E., Stults, C.L.M., and Macher, B.A. Man, apes, and old world monkeys differ from other mammals in the expression of $\alpha$-galactosyl epitopes on nucleated cells. J Biol Chem 263, 17755, 1988.

24. Oriol, R., Candelier, J.J., Taniguchi, S., Balanzino, L., Peters, L., Niekrasz, M., et al. Major carbohydrate epitopes in tissues of domestic and African wild animals of potential interest for xenotransplantation research. Xenotransplantation 6, 79, 1999.

25. Galili, U., Anaraki, F., Thall, A., Hill-Black, C., and Radic, M. One percent of circulating B lymphocytes are capable of producing the natural anti-Gal antibody. Blood 82, 248, 1993.

26. Galili, U., Mandrell, R.E., Hamadeh, R.M., Shohet, S.B., and Griffis, J.M. Interaction between human natural anti- $\alpha-$ galactosyl immunoglobulin $\mathrm{G}$ and bacteria of the human flora. Infect Immun 56, 1730, 1988.

27. Posekany, K.J., Pittman H.K., Bradfield, J.F., Haisch, C.E., and Verbanac, K.M. Induction of cytolytic anti-Gal antibodies in $\alpha$-1,3-galactosyltransferase gene knockout mice by oral inoculation with Escherichia coli O86:B7 bacteria. Infect Immun 70, 6215, 2002.

28. Stone, K.R., Abdel-Motal, U.M., Walgenbach, A.W., Turek, T.J., and Galili, U. Replacement of human anterior cruciate ligaments with pig ligaments: a model for anti- 
non-gal antibody response in long-term xenotransplantation. Transplantation 83, 211, 2007.

29. Badylak, S.F., and Gilbert, T.W. Immune response to biologic scaffold materials. Semin Immunol 20, 109, 2008.

30. Xu, H., Wan, H., Zuo, W., Sun, W., Owens, R.T., Harper, J.R., et al. A porcine-derived acellular dermal scaffold that supports soft tissue regeneration: removal of terminal galactose- $\alpha-(1,3)$-galactose and retention of matrix structure. Tissue Eng Part A 15, 1807, 2009.

31. Stone, K.R., Walgenbach, A.W., Turek, T.J., Somers, D.L., Wicomb, W., and Galili, U. Anterior cruciate ligament reconstruction with a porcine xenograft: a serologic, histologic, and biomechanical study in primates. Arthroscopy 23, 411, 2007.

32. Galili, U., Tibell, A., Samuelsson, B., Rydberg, B., and Groth, C.G. Increased anti-Gal activity in diabetic patients transplanted with fetal porcine islet cell clusters. Transplantation 59, 1549, 1995.

33. Galili, U., Chen, Z.C., Tanemura, M., Seregina, T., and Link, C.J. Induced antibody response in xenograft recipients. GRAFT 4, 32, 2001.

34. Wigglesworth, K.M., Racki, W.J., Mishra, R., SzomolanyiTsuda, E., Greiner, D.L., and Galili, U. Rapid recruitment and activation of macrophages by anti-Gal/ $\alpha$-gal liposome interaction accelerates wound healing. J Immunol 186, 4422, 2011.

35. Klos, A., Wende, E., Wareham, K.J., and Monk, P.N. International Union of Pharmacology. LXXXVII. Complement peptide C5a, C4a, and C3a receptors. Pharmacol Rev 65, 500, 2013.

36. Ravetch, J.V., and Bolland, S. IgG Fc receptors. Annu Rev Immunol 19, 275, 2001.

37. Galili, U. Interaction of the natural anti-Gal antibody with $\alpha$ galactosyl epitopes: a major obstacle for xenotranplantation in humans. Immunol Today 14, 480, 1993.

38. van Lookeren Campagne, M., Wiesmann, C., and Brown, E.J. Macrophage complement receptors and pathogen clearance. Cell Microbiol 9, 2095, 2007.

39. Valentin, J.E., Stewart-Akers, A.M., Gilbert, T.W., and Badylak S.F. Macrophage participation in the degradation and remodeling of extracellular matrix scaffolds. Tissue Eng Part A 15, 1687, 2009.

40. Stone, K.R., Walgenbach, A.W., Abrams, T., Nelson, J., Gellett, N., and Galili, U. Porcine and bovine cartilage transplants in cynomolgus monkey: I. A model for chronic xenograft rejection. Transplantation 63, 640, 1997.

41. Galili, U., LaTemple, D.C., Walgenbach, A.W., and Stone, K.R. Porcine and bovine cartilage transplants in cynomolgus monkey: II. Changes in anti-Gal response during chronic rejection. Transplantation 63, 646, 1997.

42. Stone, K.R., Ayala, G., Goldstein, J., Hurst, R., Walgenbach, A.W., and Galili, U. Porcine cartilage transplants in cyno- molgus monkey: III. Transplantation of $\alpha$-galactosidase treated porcine cartilage. Transplantation 65, 1577, 1998.

43. McPherson, T.B., Liang, H., Record, R.D., and Badylak. S.F. Gal $\alpha(1,3)$ Gal epitope in porcine small intestinal submucosa. Tissue Eng 6, 233, 2000.

44. Raeder, R.H., Badylak, S.F., Sheehan, C., Kallakury, B., and Metzger, D.W. Natural anti-galactose $\alpha 1,3$ galactose antibodies delay, but do not prevent the acceptance of extracellular matrix xenografts. Transpl Immunol 10, 15, 2002.

45. Zhu, A., and Goldstein, J. Cloning and functional expression of a cDNA encoding coffee bean $\alpha$-galactosidase. Gene 140, 227, 1994.

46. Zhu, A., Monahan, C., Zhang, Z., Hurst, R., Leng, L., and Goldstein, J. High-level expression and purification of coffee bean $\alpha$-galactosidase produced in the yeast Pichia pastoris. Arch Biochem Biophys 324, 65, 1995.

47. Butany, J., Yu, W., Silver, M.D., and David, T.E. Morphologic findings in explanted Hancock II porcine bioprostheses. J Heart Valve Dis 8, 4, 1999.

48. Cicha, I. Rüffer, A., Cesnjevar, R., Glöckler, R., Agaimy, M., Daniel, W.G. et al. Early obstruction of decellularized xenogeneic valves in pediatric patients: involvement of inflammatory and fibroproliferative processes. Cardiovasc Pathol 20, 222, 2011.

49. Clancy W.G., Narechania, R.J, Rosenberg, T.D., Gmeiner, J.G, Wisnefske, D.D., and Lange, T.A. Anterior and posterior cruciate ligament reconstruction in rhesus monkeys. J Bone Joint Surg 63, 1270, 1981.

50. Butler, D.L., Grood, E.S., Noyes, F.R., Olmstead, M.L., Hohn, R.B., Arnoczky, S.P., and Siegel, M.G. Mechanical properties of primate vascularized vs. nonvascularized patellar tendon grafts: changes over time. J Orthopedic Res 7, 68, 1989.

51. Ballock, R.T., Woo, S.L., Lyon, L.M., Hollis, J.M., and Akeson, W.H. Use of patellar tendon autograft for anterior cruciate ligament reconstruction in the rabbit: a long-term histologic and biomechanical study. J Orthopedic Res 7, 474, 1989.

52. Schindhelm, K., Rogers, G.J., Milthorpe, B.K., Hall, P.J., Howlett, C.R., Sekel, R., et al. Autograft and Leeds-Keio reconstructions of the ovine anterior cruciate ligament. Clin Orthop Relat Res 267, 278, 1991.

Address correspondence to: Uri Galili, PhD UMass Medical School (retired) 910 S. Michigan Ave. Apt. 1404 Chicago, IL 60605

E-mail: uri.galili@rcn.com

Received: August 9, 2016

Accepted: January 6, 2017

Online Publication Date: February 14, 2017 